\title{
Endothelial cell activation in vasculitis of peripheral nerve and skeletal muscle
}

\author{
Peter K Panegyres, Randall J Faull, Graeme R Russ, Sarah L Appleton, \\ Anders G Wangel, Peter C Blumbergs
}

\begin{abstract}
To clarify the role of endothelial cells in the pathogenesis of vasculitis affecting peripheral nerve and skeletal muscle, the endothelial expression of adhesion molecules and major histocompatibility antigens (MHC) in different vasculitic syndromes were studied, and related to the presence of anti-endothelial cell antibodies (AECA). Increased expression of the intercellular adhesion molecule ICAM-1 in vasculitic lesions in nerve and muscle was shown, and this was associated with increased expression of MHC class I and II antigens. AECA were detected in low titre in only a minority of patients. The findings suggest that endothelial cells have a critical role in mediating the tissue injury in vasculitis affecting nerve and muscle and that the process is triggered by cellular and not antibody-mediated mechanism in the majority of patients.
\end{abstract}

Vascular endothelial cells are active participants in inflammatory processes. They express a number of molecules essential for cell-cell interactions, including antigens from the major histocompatibility complex (MHC) and the intercellular adhesion molecules, ICAM-1 (intercellular adhesion molecule-1) and ELAM-1 (endothelial leukocyte adhesion molecule-1). ICAM-1 is a ligand for the leukocyte adhesion molecule LFA-1 (lymphocyte function-associated antigen-1), and ELAM-1 is a mediator of neutrophil adhesion. ${ }^{1-3}$ To clarify the role of endothelial cells in the pathogenesis of vasculitis of peripheral nerve and skeletal muscle we studied the endothelial expression of these molecules in different vasculitic syndromes. We compared them to the expression in normal tissues, and in tissues from patients with noninflammatory diseases of muscle and nerve.

Antibodies directed against vascular endothelial cells have been implicated in the pathogenesis of systemic vasculitis. ${ }^{4}$ We therefore also examined sera from our patients for the presence of anti-endothelial cell antibody activity, to see if antibodies recognising endothelial cell targets may play a role in the pathogenesis of vasculitis of peripheral nerve and skeletal muscle.

\section{Materials and methods}

PATIENTS

Inflammation and necrosis of blood vessels in peripheral nerve, skeletal muscle, or both was necessary for inclusion in the study. ${ }^{5}$ The patients were selected as representative of the different clinicopathological entities we have previously defined in our studies of the immunopathic mechanisms of vasculitis affecting peripheral nerve and skeletal muscle. ${ }^{6}$

\section{Monoclonal antibodies}

The following were used: WEHI-CAM-1: an $\mathrm{IgG}_{2} \mathrm{~b}$ murine monoclonal antibody directed against intercellular adhesion molecule-1 (ICAM-1); was donated by Dr A Boyd, Walter and Eliza Hall Institute, Melbourne, Australia; ${ }^{7}$ 1.2B6: an $\mathrm{IgG}_{1}$ antibody directed against endothelial leukocyte adhesion molecule -1 (ELAM-1); was donated by Dr D Haskard, Guy's Hospital, London; ${ }^{8}$ W6/32: an $\mathrm{IgG}_{2} \mathrm{~b}$ antibody directed against HLA class I molecules; RM 5.112: an $\operatorname{IgG}_{2}$ a antibody directed against a monomorphic epitope of HLA class II molecules was produced in the laboratory of Dr A d'Apice, Royal Melbourne Hospital, Australia; 1D4.5: an $\operatorname{IgG}_{2}$ a antibody with no known reactivity with human tissue and used as a negative control.

\section{INDIRECT IMMUNOPEROXIDASE STAINING}

Frozen sections of muscle and peripheral nerve biopsies were stained as previously described. ${ }^{9}$ The sections were all stained on the same day and read "blind" by two people. Triplicate slides were examined for each stain and were concordant. The intensity of reaction product was assessed semiquantitatively and graded from - through to ++++ .

ANTI-ENDOTHELIAL CELL ANTIBODY

(AECA) ASSAY

Human umbilical vein endothelial cells were cultured according to the methods of Jaffe $e t$ $a_{l}{ }^{10}$ Cells were seeded into sterile 96 well microtitreplates, grown until confluent, and fixed with $0.2 \%$ gluteraldehyde for five minutes. For some experiments the cells were stimulated with recombinant interleukin 1 (Boehringer, Mannheim, Germany), 5 units/ $\mathrm{ml}$ for four hours.

AECA were assayed by an enzyme linked immunosorbent assay (ELISA). Wells were blocked with $10 \%$ newborn calf serum in phosphate buffered saline (PBS) for one hour at room temperature. Patients' sera were diluted $1 / 200$ in washing buffer (PBS/0.2\% bovine serum albumin), and $100 \mu \mathrm{l}$ were added to each well and left to incubate for two hours at room temperature. They were tested in triplicate. Wells were washed three times in 
Table ICAM-1, ELAM-1 and MHC expression on endothelial cells

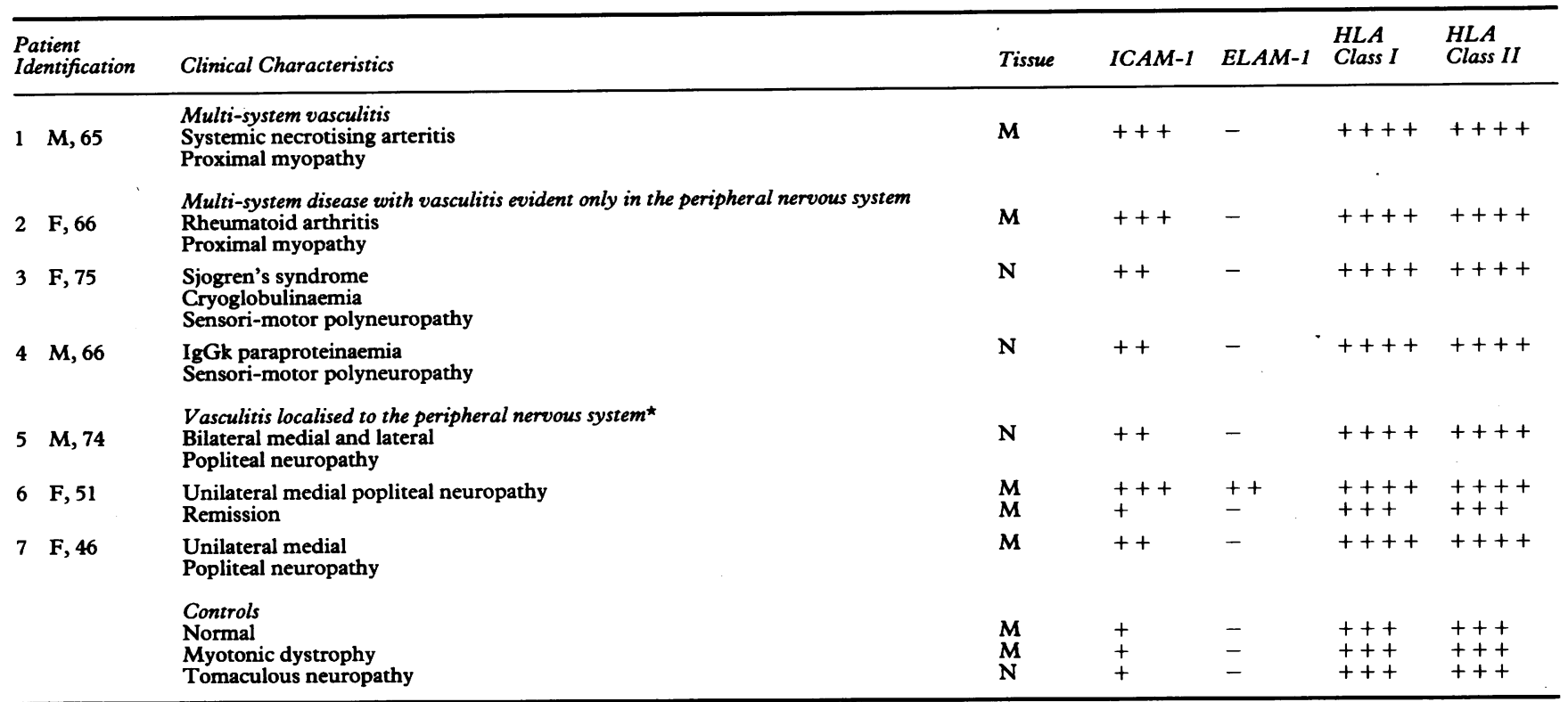

*No clinical or laboratory evidence of multisystem disease or vasculitis.

M = Skeletal Muscle.

$\mathbf{N}=$ Peripheral Nerve.

washing buffer, and $100 \mu$ lof goat anti-human IgG (Tago USA), diluted in 1/7500 in washing buffer was added to each well for two hours at room temperature. Wells were again washed three times in washing buffer, then $100 \mu \mathrm{l}$ of substrate p-nitrophenylphosphate, $1 \mathrm{mg} / \mathrm{ml}$ in diethanolamine buffer were added to each well and incubated at $37^{\circ} \mathrm{C}$. Absorbance was read at 405 and $540 \mathrm{~nm}$. Absorbance values greater than 0.3 were regarded as positive (mean plus three standard deviations of 80 normal subjects).

\section{Results}

The clinical features of patients and the semiquantitative assessment of the endothelial staining of each biopsy is summarised in the table.

The endothelial expression of each antigen was very similar in the three control tissues examined (normal muscle, muscle from a patient with myotonic dystrophy, and nerve from a patient with tomaculous neuropathy). The latter two were chosen because inflammation is not a feature of the pathology. In these uninflamed tissues ICAM-1 is weakly expressed on the intima of large vessels and on capillary endothelium, but ELAM-1 is uniformly absent. HLA class $I$ is moderately expressed on the intima of large vessels and on capillaries, and is also found in small amounts in the wall of large vessels. HLA class II is confined to the endothelial cells and is generally slightly weaker than HLA class I.

Patient 6 gave us the best opportunity to compare the expression of these antigens in vasculitis. A muscle biopsy taken from this patient during remission was virtually indistinguishable from normal muscle both morphologically and with respect to the expression of ICAM-1, ELAM-1, and HLA class I and class
II antigens. A biopsy taken at diagnosis of the vasculitis showed an intense perivascular inflammatory infiltrate which was largely composed of macrophages and lymphocytes, and some neutrophils. The magnitude of this inflammatory response was greater than the biopsies from the other patients. In this biopsy there was a clear increase in the endothelial expression of ICAM-1. ELAM-1 was also definitely visible on the intima of a number of medium to large vessels located in the areas of greatest inflammatory infiltration (fig 1). HLA class I and class II antigens were also increased but this was not as dramatic. In addition the muscle fibres clearly were HLA class I positive in the vasculitic biopsy but were negative for HLA class II, a feature which was not found in any of the non-inflamed biopsies. The infiltrating leucocytes were also strongly HLA class I and class II positive.

The biopsies taken from the other patients with vasculitis show changes similar to patient 6 at diagnosis with enhanced expression of ICAM-I and HLA class I and II antigens. However, ELAM-1 was not detected in these patients.

Sera from the seven patients examined above in addition to another five patients with vasculitis of the peripheral nervous system were also assessed for the presence of anti-endothelial cell antibodies (AECA). Only patients 4 and 9 had detectable AECA, which were of low titre (fig 2).

\section{Discussion}

The endothelium in normal muscle and peripheral nerve expresses HLA class I and II antigens and ICAM-1. ELAM-1, however, is not expressed in normal tissue. In patients with vasculitis, the inflammatory reaction is associated with increased expression of these 

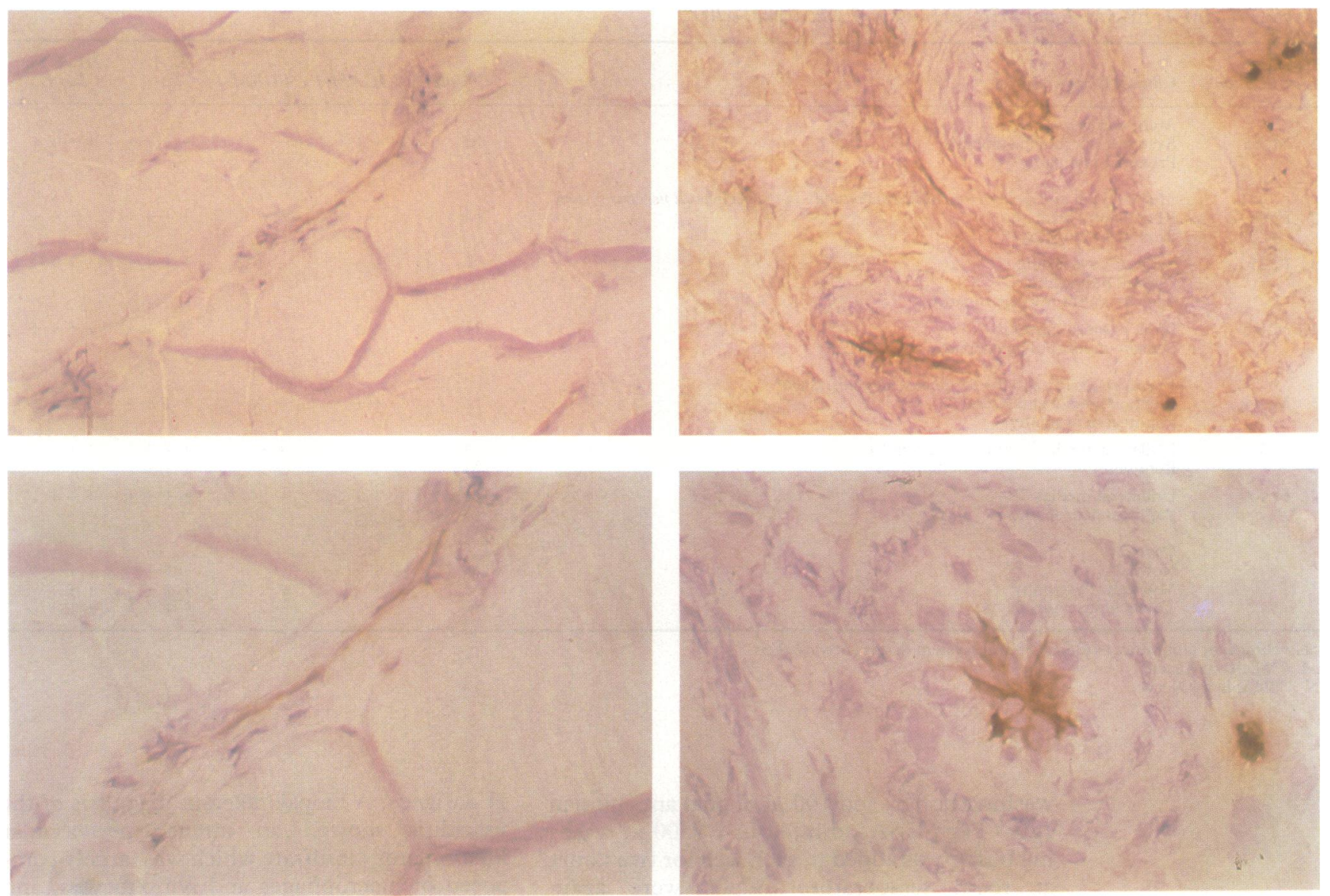

Figure 1 ICAM-1 and ELAM-1 expression in skeletal muscle at diagnosis and remission for patient 6. (Top panel for ICAM-1, left in remission, right at diagnosis $\times 300$; Bottom panel for ELAM-1, left in remission, right at diagnosis $\times 600)$.

molecules on the endothelium. This was particularly notable for ICAM-1, as its expression on endothelium in normal and uninflamed tissue is low.

Patient 6 offered us the greatest opportunity to study endothelial activation, as tissue was available not only at diagnosis, but also during remission. This patient had increased expres-

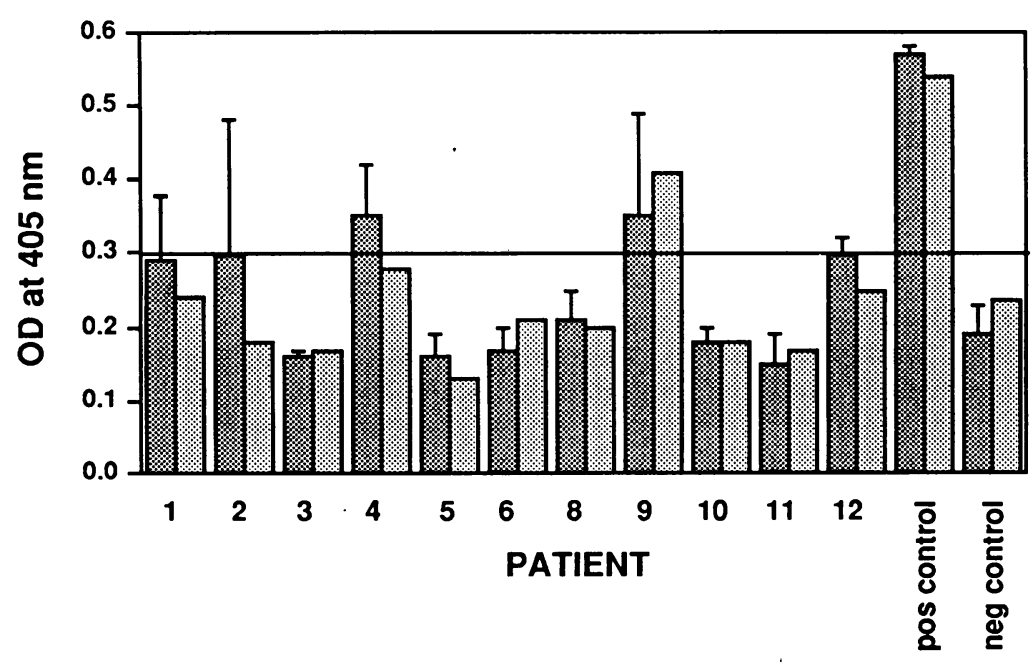

Figure 2 Anti-endothelial cell antibodies in patients with vasculitis affecting nerve and muscle. Patient identification as for the table. Patient $8 \mathrm{~F} / 70$ rheumatoid arthritis and peripheral neuropathy; patient 9 F/51 nonsystemic vasculitis with a mononeuropathy; patient $10 \mathrm{~F} / 79$ nonsystemic vasculitis with multiple mononeuropathy; patient $11 \mathrm{M} / 72$ rheumatoid arthritis with peripheral neuropathy; patient $12 \mathrm{~F} / 62$ rheumatoid arthritis with mononeuropathy. (Dark hatch = unstimulated endothelial cells; light hatch = interleukin-1 stimulated endothelial cells. Laboratory cut-off is an optical density (OD) of 0.3 at $405 \mathrm{~nm}$ ). sion of ELAM-1, as well as ICAM-1, and HLA class I and class II. The ELAM-1 expression was identified in medium to large vessels in the areas of greatest inflammatory reaction. The expression of ELAM-1 in this patient, but not in the others, is most likely to be a result of the timing of the biopsy in relation to the onset of vasculitis, as ELAM-1 usually disappears in in vitro activation after 24 hours despite continued stimulation. ${ }^{11}$ The possibility also exists that the release of cytokines was greatest in this patient because of the greater intensity of the inflammatory reaction.

In vitro studies with cultured endothelial cells have shown that HLA class I, II, ICAM-1 and ELAM-1 are all increased after stimulation with cytokines such as interleukin 1, tumour necrosis factor, and $\gamma$-interferon. ${ }^{11-13}$ We postulate that the activated infiltrating inflammatory cells in vasculitis secrete such cytokines, with the consequent increased expression of these antigens.

These antigens have important roles in cellcell interactions in inflammatory responses and we postulate the following mechanism in vasculitis of the peripheral nervous system: unknown antigen is presented with HLA class II molecules by endothelial cells to helper T-cells. This interaction is stabilised by adhesion molecules on the T-cell (LFA-1) and its ligand on endothelial cells ICAM-1. The endothelial cells are stimulated to release interleukin-1 which in turn stimulates the upregulation of HLA class I and ICAM-1 on adjacent endo- 
thelial cells. The helper T-cells release $\gamma$-interferon which upregulates HLA class II molecules, and interleukin-2 which induces proliferation of other antigen-specific T-lymphocytes. More helper T-cells bind MHC class II molecules plus antigen on endothelial cells. A cellular cascade results in the accumulation of a T-lymphocyte population with specificity directed toward the initiating antigen. This is followed by cellular migration into the surrounding tissues and tissue damage.

The increased expression of adhesion molecules and MHC antigens shows that vascular endothelial cells in vasculitis affecting the peripheral nervous system react in a similar manner to those in other inflammatory states, such as allograft rejection and Kawasaki disease. $^{1}$

Only two patients out of 12 had detectable AECA, and the titre was low compared with those found in other autoimmune diseases such as systemic lupus erythematosus (S Appleton, unpublished observations). These antibodies probably have little role in initiating the pathogenic reactions of vasculitis involving muscle and nerve, but they may be important in systemic vasculitic syndromes. ${ }^{4}$

In conclusion, we have shown that the endothelium expresses increased amounts of HLA class I and II antigens, ICAM-1, and sometimes ELAM-1 in vasculitis within muscle and nerve. Endothelial cells therefore play a fundamental role in the pathogenesis of vasculitis affecting the peripheral nervous system. Their activiation shows that they form part of the pathway through which tissue injury is mediated. Our results suggest a potential therapeutic role for monoclonal antibodies directed against endothelial antigens in those patients who do not respond to conventional immunosuppressive treatment.

1 Cotran RS, Pober JS. Effects of cytokines on vascular endothelium: their role in vascular and immune injury. Kidney Int 1989;35:969-75.

2 Hynes RO. Integrins: a family of cell surface receptors. Cell 1987;48:549-54.

3 Wawryk SO, Novotny JR, Wicks IP, et al. The role of the LFA-1/ICAM-1 interaction in human leukocyte homing and adhesion. Immunol Rev 1989;108:135-61.

4 Brasile L, Kremer JM, Clarke JL, Cerilli J. Identification of an autoantibody to vascular endothelial cell-specific antigens in patients with systemic vasculitis. $\mathrm{Am} \mathrm{J} \mathrm{Med}$ 1989;87:74-80.

5 Fauci AS. The spectrum of vasculitis. Clinical, pathological, immunologic, and therapeutic considerations. Ann Intern Med 1978;89(P1):660-76.

6 Panegyres PK, Blumbergs PC, Leong A S-Y, Bourne AJ. Vasculitis of Peripheral Nerve and Skeletal Muscle: Clinicopathological Correlation and Immunopathic Mechanisms. INeurol Sci 1990;100:193-202.

7 Boyd AW, Wawryk SO, Burns GF, Fecondo JV. Intercellular adhesion molecule-1 (ICAM-1) has a central role in cell-cell contact-mediated immune mechanisms. Proc in cell-cell contact-mediated immune

8 Wellicome SM, Thornhill MH, Pitzalis C, et al. A Monoclonal Antibody that detects a novel antigen on Endothelial cells that is induced by Tumour Necrosis Factor, IL1, or Lipopolysaccharide. J Immunol 1990;144:2558-65.

9 Faull RJ, Russ GR. Tubular expression of intercellular adhesion molecule-1 during renal allograft rejection. Transplantation 1989;48:226-30.

10 Jaffe EA, Nachman RL, Becker CG, Minick CR. Culture of human endothelial cells derived from umbilical veins: identification by morphologic and immunologic criteria. $J$ Clin Inves 1973;52:2745-56.

11 Pober JS, Cotran RS. Cytokines and Endothelial Cell Biology. Physiol Rev 1990;70:427-51.

12 Collins T, Korman AJ, Wake CT, et al. Immune interferon activates multiple class II major histocompatibility complex genes and the associated invariant chain in human endothelial cells and dermal fibroblasts. Proc Natl Acad Sci (USA) 1984;81:4917-2

13 Mantovani A, Dejana E. Cytokines as communication signals between leukocytes and endothelial cells. Immunol Today 1989;10:370-5. 